\title{
On mathematical modelling of complex ecosystems: Application to marine planktonic patchiness
}

\author{
D. M. Dubois \\ Université de Liège, Institut de Mathématique; Liège, Belgique
}

\begin{abstract}
Mathematical modeling of complex ecosystems is very difficult due to the very large number of components in the real ecosystem. Conceptual subdivision into interacting sub-systems is necessarily subjective and is made in view of explaining a particular aspect of the reality. In this paper, the North Sea planktonic ecosystem is reduced to a rather simple mathematical model with the purpose of showing the possibility of a spontaneous spatial emergence of plankton patches by diffusive instability. Due to the dependence of diffusion coefficients on the differential diameters of phytoplankton and herbivorous zooplankton patches, respectively, the spatially homogeneous steady state is unstable for spatial perturbations with wavelengths belonging to a certain range of values. As a consequence, these perturbations amplify leading to spatial heterogeneity.
\end{abstract}

\section{INTRODUCTION}

Mathematical modelling of complex ecosystems is very difficult due to the very large number of components in the real ecosystem. Conceptual subdivision of a real ecosystem into interacting sub-systems is necessarily subjective. Moreover, this subdivision is always made to attempt to explain a particular phenomenon.

I will focus attention on the mathematical modelling of the mechanism of patches emergence. occurring in the marine planktonic ecosystem. A recent review on this field has been presented by Levin (1976). References dealing with some biological processes in the whole planktonic ecosystem are given by Dubois \& Mayzaud (1976).

The general problem dealing with non-homogenous spatial pattern is of great interest in many other fields. On the one hand, morphogenesis related to structural stability was studied by Thom $(1972,1974)$. On the other hand, morphogenesis in relation with diffusive instability was firstly developed by Turing (1952) and applied largely by others (e.g. Glansdorff \& Prigogine, 1974).

In this paper, some ideas about patchiness have been inspired by these theories; more details about model systems of ecological morphogenesis can be found in Dubois (1976b) and Dubois \& Closset (1976). 


\section{RESULTS}

\section{Modelling of ecosystems}

An ecological system can be defined as a system for which a flow of energy and matter from its environment is a necessary condition to the maintenance of its functionality. Contrary to classical physical systems which are described in the energy space, ecological systems must be described, not only in the energy-space, but also in the energy and matter flow-space.

At the level of energy, a structural stability will be defined, while at the level of energy and matter flow, a functional stability will be studied. The flow-space will be the supplementary dimension for describing the logic of living systems, for the purpose of understanding their functionality.

Emergence of structural and functional properties of ecological systems will be the consequence of exchanges of energy and matter between the components of a system and between the system and its environment. In the real world, the dynamics of phenomena depends on such a large number of variables and parameters at all spatial and temporal scales that the observer is unable to obtain a global view of the reality. For describing, quantitatively, the evolution of an open system, the observer will build a mathematical model of energetic processes occurring in the system.

For that purpose, the observer subdivides the system into a certain number of components. In these conditions, the mathematical model will only be a particular representation of the reality. Thus, necessarily, an uncertainty of the exact knowledge of the real world will exist for the observer.

From experimental data, the observer subdivides a system into interacting subsystems. This subdivision process leads to a rather subjective understanding of the reality. The observer will then define emettors, receptors and communication channels of energy and matter inside the system and between the system and its environment.

After this step, the observer will build a particular model with a purpose in his mind: the explanation of mechanisms dealing with the studied system.

\section{On mathematical modelling}

From a very general point of view, let us consider a system of volume $\mathrm{V}$ enclosed by a surface of area $A$. If the concentration of any component $i$ par unit volume at a point inside the system is denoted by $c_{i}$, the local flow vector $J_{\mathrm{i}}$ may be defined by

$$
J_{\mathrm{i}}=\mathrm{c}_{\mathrm{i}} V_{\mathrm{i}}
$$

where $V_{\mathrm{i}}$ is the geometrical translation of the ith component represented by its velocity at the given point.

For characterizing the behavior of the flow, Gauss introduced the notion of divergence of the flow:

$$
\operatorname{div} J_{\mathrm{i}} \equiv \nabla \cdot J_{\mathrm{i}}=\frac{\partial \mathrm{J}_{\mathrm{i}}(\mathrm{x})}{\partial \mathrm{x}}+\frac{\partial \mathrm{J}_{\mathrm{i}}(\mathrm{y})}{\partial \mathrm{y}}+\frac{\partial \mathrm{J}_{\mathrm{i}}(\mathrm{z})}{\partial \mathrm{z}}
$$


where $J_{i}(x), J_{i}(y)$ and $J_{i}(z)$ are the projections of the flow $J_{i}$ on the three spatial cartesian axis and $x, y$ and $z$ the spatial coordinates.

The physical meaning of $\operatorname{div} J_{\mathrm{i}}$ is the following: a positive divergence means that at the point under consideration there is a net outflow of the ith species, or, in more descriptive language, the point of positive divergence is a "source" of species i. On the other hand, a negative divergence indicates a net inflow of the ith species; the point under consideration is a " $\operatorname{sink}$ ". At points where $\operatorname{div} J_{\mathrm{i}}=\mathrm{o}$, there is neither accumulation nor removal of material.

Gauss demonstrated that the integral of the divergence over the volume is equal to the total flow of the ith species through the surface bounding the volume:

$$
\int_{\mathrm{V}} \nabla \cdot J_{\mathrm{i}} \mathrm{dV}=\int_{\mathrm{A}} J_{\mathrm{i}} \cdot \mathrm{d} A
$$

The concept of divergence is very useful in the consideration of local conservation laws at all points of a system.

It can be shown that for non-conservative continuous systems, the local conservation law of $c_{i}$ is given by

$$
\frac{\partial c_{i}}{\partial t}=I_{i}-\nabla \cdot J_{i}
$$

where $I_{i}$ is the local expression for the transformation or formation of species $i$ as a consequence of chemical, biological interactions with other species. The difficulty is to find the best mathematical formulation for both flow and interaction terms.

\section{Modelling the mechanism of the spatial emergence of plankton patches}

Let us consider the following sub-ecosystem which is subdivided into two components: the phytoplankton and the herbivorous zooplankton populations. This sub-ecosystem is in interaction (feed-back) with its environment, i.e. the remaining ecosystem (nutrients, bacteria, non-living particulate matter, omnivorous, carnivorous, etc.) and the physical environment (seawater, light, etc.).

The general mathematical model is written:

$$
\begin{aligned}
& \frac{\partial N_{1}}{\partial t}=f_{1}\left(N_{1}\right) N_{1}-f_{2}\left(N_{1}\right) N_{1} N_{2}+\nabla \cdot\left(K_{1} \nabla N_{1}\right) \\
& \frac{\partial N_{2}}{\partial t}=-f_{3}\left(N_{2}\right) N_{2}+f_{4}\left(N_{1}\right) N_{1} N_{2}+\nabla \cdot\left(\mathrm{K}_{2} \nabla N_{2}\right)
\end{aligned}
$$

where $\mathrm{N}_{1}$ and $\mathrm{N}_{2}$ represent the phytoplankton and the herbivorous zooplankton concentrations, respectively; and $\mathrm{K}_{1}$ and $\mathrm{K}_{2}$ are the diffusivity coefficients of the phytoplankton and herbivorous zooplankton inside a patch respectively. In this study horizontal variability will be taken into account. In comparing equations (2) to (4), one can see that the mathematical expression of the local flow vector $J_{\mathrm{i}}$ of population $\mathrm{i}$ is given by

$$
J_{\mathrm{i}}=-\mathrm{K}_{\mathrm{i}} \nabla \mathrm{N}_{\mathrm{i}} \equiv-\mathrm{K}_{\mathrm{i}} \operatorname{grad} \mathrm{N}_{\mathrm{i}}
$$

which depends on the gradient (grad.) of the concentration of the population $\left(\mathrm{N}_{\mathrm{i}}\right)$ with a factor of proportionality given by the diffusion coefficient $\left(\mathrm{K}_{\mathrm{i}}\right)$. 
Interest is directed to the following spatially homogeneous steady state

$$
\begin{gathered}
\left(\partial \mathrm{N}_{1} / \partial t=\partial \mathrm{N}_{2} / \partial t=0\right) \\
\mathrm{N}_{10}=\frac{f_{3}\left(\mathrm{~N}_{20}\right)}{f_{4}\left(\mathrm{~N}_{10}\right)} \\
\mathrm{N}_{20}=\frac{f_{1}\left(\mathrm{~N}_{10}\right)}{f_{2}\left(\mathrm{~N}_{10}\right)}
\end{gathered}
$$

which give the classical non-trivial spatially homogeneous steady state (e.g. Dubois, 1976a) $\mathrm{N}_{10}=\mathrm{k}_{3} / \mathrm{k}_{4}$ and $\mathrm{N}_{20}=\mathrm{k}_{1} / \mathrm{k}_{2}$ in the classical Lotka-Volterra prey-predatormodel.

In the next section, it will be shown mathematically that, under certain conditions, the spatially homogeneous steady state can be unstable for some well-defined spatial perturbations. This local instability is due to non-equal diffusivities $K_{1} \neq K_{2}$ of the phytoplankton and herbivorous zooplankton populations, respectively, leading to the emergence of plankton patches with well-defined diameters $\varnothing_{1}$ and $\varnothing_{2}$ satisfying $\mathrm{K}_{1}=\mathrm{K}\left(\varnothing_{1}\right)$ and $\mathrm{K}_{2}=\mathrm{K}\left(\varnothing_{2}\right)$.

The physical mechanism of the spatial emergence of plankton patches can be explained as follows.

Let me consider the spatially homogeneous steady state. The planktonic ecosystem will be stable locally if spatial heterogeneities created by random perturbations around the spatial homogeneous steady state regress with time. As shown elsewhere (Dubois, 1975; Dubois \& Adam, 1976), the phytoplankton behaves as an activator and the herbivorous zooplankton as an inhibitor in the creation of what we called an ecological prey-predator wave. For values of the wavelength of perturbations belonging to a certain range around a critical wavelength $\lambda_{\mathrm{c}}$ (see the mathematical expression in section 4) these perturbations will amplify. Indeed, in the real ecosystem, the diffusivity of seawater is not constant but increases with the diameter $\varnothing$ of the seawater patch. If after some random perturbations, the spatial repartition of plankton has characteristic lengths given by $\varnothing_{1}$ and $\varnothing_{2}$ for the phytoplankton and herbivorous zooplankton populations respectively, the damping effect for the phytoplankton spatial inhomogeneity will be smaller than for the herbivorous zooplankton. In these conditions, the activator (the phytoplankton) will posses a small damping effect and the inhibitor (the herbivorous zooplankton) will posses a great damping effect. And, for some conditions of non-linear ecological interactions between the activator and the inhibitor, due to the faster diffusion of the inhibitor, the activator will amplify its activating effect leading to the formation of a phytoplankton patch. With a certain time lag, an inhomogeneity will appear in the herbivorous zooplankton spatial repartition and we will then assist to the spatial structuration of a plankton patch.

The horizontal pattern of the patches repartition will be given by an hexagonal symmetry in ideal conditions, i.e. without environmental large disturbances like advection currents. This hexagonal repartition is logic from the point of view of the optimization of the spatial occupancy. Moreover, patches can differ from each other in their species content: it should exist of a competitive exclusion principle between patches which then would play the role of planktonic niches. 
Mathematicaldemonstration of the diffusive instability in the planktonic model system

Let us now consider small perturbations $n_{1}$ and $n_{2}$ around the homogeneous steady state (eqs. [6] and [7])

$$
\begin{aligned}
& N_{1}=N_{10}+n_{1} \\
& N_{2}=N_{20}+n_{2}
\end{aligned}
$$

substitution of eqs. (8) and (9) into 3 and 4 leads to, after linearization,

$$
\begin{aligned}
& \frac{\partial \mathrm{n}_{1}}{\partial \mathrm{t}}=\mathrm{a}_{11} \mathrm{n}_{1}+\mathrm{a}_{12 \mathrm{n}_{2}}+\mathrm{K}\left(\phi_{1}\right) \nabla^{\mathrm{z}_{\mathrm{n}_{1}}} \\
& \frac{\partial \mathrm{n}_{2}}{\partial \mathrm{t}}=\mathrm{a}_{21} \mathrm{n}_{1}+\mathrm{a}_{22 \mathrm{n}_{2}}+\mathrm{K}\left(\phi_{2}\right) \nabla^{\mathbf{a}_{2}}
\end{aligned}
$$

where diameters (which are estimated by the variances of the spatial distributions of populations) of phytoplankton and herbivorous zooplankton patches are fixed and given by $\varnothing_{1}$ and $\varnothing_{2}$ (this assumption is correct during the first phase of the patches emergence). The coefficients $a_{i j}$ are given by

$$
\begin{gathered}
a_{11}=f_{10}-f_{20} N_{20}-f_{20}^{\prime} N_{10} N_{20}+f_{10}^{\prime} N_{10} \\
a_{22}=f_{40} N_{10}-f_{30}-f_{30}^{\prime} N_{20} \\
a_{12}=-f_{20} N_{10} \\
a_{21}=f_{40} N_{20}+f_{40}^{\prime} N_{10} N_{20}
\end{gathered}
$$

where $f_{10}=f_{1}\left(N_{10}\right), f_{20}=f_{2}\left(N_{10}\right), f_{30}=f_{3}\left(N_{20}\right), f_{40}=f_{4}\left(N_{10}\right)$, and $f_{10}^{\prime}=$ $\left(\partial \mathrm{f}_{1} / \partial \mathrm{N}_{1}\right)_{0}, \mathrm{f}^{\prime} \mathrm{f}_{00}=\left(\partial \mathrm{f}_{2} / \partial \mathrm{N}_{1}\right)_{0}, \mathrm{f}_{30}^{\prime}=\left(\partial \mathrm{f}_{3} / \partial \mathrm{N}_{2}\right)_{0}$ and $\mathrm{f}_{40}^{\prime}=\left(\partial \mathrm{f}_{4} / \partial \mathrm{N}_{1}\right)_{0}$.

To know whether the homogeneous steady state is stable it is sufficient to study the behavior of solutions of equations (10) and (11) which have the form (Fourier's analysis)

$$
\mathrm{n}_{\mathrm{i}}(\mathrm{x}, \mathrm{y}, \mathrm{t})=\tilde{\mathrm{n}}_{\mathrm{i}} \cos (k \cdot r+\phi) \exp (\sigma \mathrm{t}) \quad(\mathrm{i}=1,2)
$$

where $\tilde{n}_{\mathrm{i}}, k, \phi$ and $\sigma$ are constants. Stability is assured if and only if all these solutions decay with time, i.e. if and only if $\sigma$ has a negative real part. It will take place if and only if the two following conditions hold

$$
\begin{gathered}
\left(\mathrm{a}_{11}-\mathrm{K}\left(\phi_{1}\right) \mathrm{k}^{2}\right)+\left(\mathrm{a}_{22}-\mathrm{K}\left(\phi_{2}\right) \mathrm{k}^{2}\right)<0 \\
\left(\mathrm{a}_{11}-\mathrm{K}\left(\phi_{1}\right) \mathrm{k}^{2}\right)\left(\mathrm{a}_{22}-\mathrm{K}\left(\phi_{2}\right) \mathrm{k}^{2}\right)-\mathrm{a}_{12} \mathrm{a}_{21}<0
\end{gathered}
$$

For having diffusive instability (Turing, 1952), perturbations of zero wavenumber are required to be stable (global stability of the ecosystem). For $\mathrm{k}=0$, eqs. (17) and (18) give necessary conditions for diffusive instability,

$$
\begin{gathered}
a_{11}+a_{22}<0 \\
a_{11} a_{22}-a_{12} a_{21}<0
\end{gathered}
$$

Equations (17) is always verified for all positive values of $\mathrm{k}^{2}$, meanwhile eq. (18) 
can be violated for finite positive real values of $\mathrm{k}^{2}$. The roots $\mathrm{k}^{2}$ solutions of eq. (18) equal to zero,

$$
\left(\mathrm{a}_{11}-\mathrm{K}\left(\phi_{1}\right) \mathrm{k}^{2}\right)\left(\mathrm{a}_{22}-\mathrm{K}\left(\phi_{2}\right) \mathrm{k}^{2}\right)-\mathrm{a}_{12} \mathrm{a}_{21}=0
$$

are given by

$$
\mathrm{k}^{2}=\frac{\mathrm{a}_{11} \mathrm{~K}\left(\phi_{2}\right)+\mathrm{a}_{22} \mathrm{~K}\left(\phi_{1}\right) \pm \sqrt{\left(\mathrm{a}_{11} \mathrm{~K}\left(\phi_{2}\right)+\mathrm{a}_{22} \mathrm{~K}\left(\phi_{1}\right)\right)^{2}-4 \mathrm{~K}\left(\phi_{1}\right) \mathrm{K}\left(\phi_{2}\right)\left(\mathrm{a}_{11} \mathbf{a}_{22}-\mathrm{a}_{12} \mathrm{a}_{21}\right)}}{2 \mathrm{~K}\left(\phi_{1}\right) \mathrm{K}\left(\phi_{2}\right)}
$$

For values of $\mathrm{k}^{2}$ between these two roots, eq. (21) is negative thus stability condition (18) is violated, with the conditions

and

$$
\mathrm{a}_{11} \mathrm{~K}\left(\phi_{2}\right)+\mathrm{a}_{22} \mathrm{~K}\left(\phi_{1}\right)>0
$$

$$
\left(a_{11} \mathrm{~K}\left(\phi_{2}\right)+\mathrm{a}_{22} \mathrm{~K}\left(\phi_{1}\right)\right)^{2} \geqq 4 \mathrm{~K}\left(\phi_{1}\right) \mathrm{K}\left(\phi_{2}\right)\left(\mathrm{a}_{11} \mathrm{a}_{22}-\mathrm{a}_{12 \mathrm{a}_{21}}\right)
$$

for having real and positive values of $\mathrm{k}^{2}$. When equality occurs in the condition 24 , we obtain a double root. In this case, only one wavenumber corresponding to a critical wavelength $\lambda_{c}$ is unstable

$$
\lambda_{\mathrm{e}}=\frac{2 \pi}{\mathrm{k}_{\mathrm{c}}}=2 \pi \sqrt{\frac{2 \mathrm{~K}\left(\phi_{1}\right) \mathrm{K}\left(\phi_{2}\right)}{\mathrm{a}_{11} \mathrm{~K}\left(\phi_{2}\right)+\mathrm{a}_{22} \mathrm{~K}\left(\phi_{1}\right)}}
$$

\section{DISCUSSION}

I wanted to show that, even in a spatially homogeneous environment, spontaneously the planktonic model system exhibits instabilities for spatial fluctuations belonging to a certain range of wavelengths.

The solution of the equations of my model system gives a spatial heterogeneity of plankton repartition as a consequence of fundamental mechanisms occurring in the real ecosystem. My approach to mathematical modelling involves the construction of a model system by selecting the main phenomena and by taking into account only wellknown experimental facts. However, the modelling technique employed is different from most usual techniques presented thus far in the scientific literature.

Some authors attempt to build a sophisticated mathematical model in adjusting the parameters with experimental curves. Others, knowing that the spatial repartition of plankton is heterogeneous, search for mathematical models which give solutions in the form of heterogeneity of plankton repartition, even if their equations do not reflect some well-known experimental results.

Many models thus led to unrealistic conclusions about the mechanism of the real phenomenon. In fact, most sophisticated mathematical models can give practically any solutions by adjusting the parameters. The mathematical model of a particular mechanism must be the more simple one and compatible with available data: this technique can be called the dynamical reductionism.

Our model of the emergence of plankton patches is the simpler model $(2 \mathrm{com}-$ ponents) which could be constructed in taking into account the less drastic constraint on the mathematical expression of the ecological interactions.

Obviously, to obtain a more realistic model which could give quantitative solu- 
tions closer to experimental data, it is necessary to sophisticate the model by taking into account more phenomena like (a) the horizontal distribution of nutrients which modify the growth of phytoplankton from point to point in the sea; (b) the upwelling effect which is open of great importance (in the North Sea, this effect can be neglected); (c) the turbidity of seawater which will also modulate the spatial pattern in changing the extinction coefficient from point to point; (d) the light variation during the year; (e) seawater transport properties other than diffusion (e.g. convection, advection); (f) the dynamics of bacteria, non-living particulate matter, omnivorous, carnivorous, etc.

However, it is simpler to sophisticate a simple model rather than to simplify a sophisticated one. If a simple model is impossible to build for some reason, a sophisticated model is useless and will explain nothing, even if the solutions agree precisely (too much, perhaps!) with experimental curves.

\section{LITERATURE CITED}

Dubois, D. M., 1975. A model of patchiness for prey-predator plankton populations. Ecol. Modeling 1, 67-80.

- 1976a. Modelling and simulation of the mesoscale mosaic structure of the lower marine trophic levels. Springer, Berlin, 407-418 (Lecture notes in computer science. Vol. 40).

- 1976b. On temporal and spatial structure in model systems and application to ecological patchiness. In: Analyse de systèmes et ses orientations nouvelle. IRIA, 599-613.

- \& Adam, Y., 1976. Spatial structuration of diffusive prey-predator biological populations: simulation of the horizontal distribution of plankton in the North Sea. In: System simulation in water resources. Ed. by G. C. Vansteenkiste. North-Holland, Amsterdam, 343-356.

- \& Closset, P. L., 1976. Patchiness in primary and secondary production in the Southern Bight: a mathematical theory. In: Proceedings of the 10th European Symposium on Marine Biology. Ed. by G. Persoone \& E. Jaspers. University Press, Wetteren, 2, 211-229.

- \& Mayzaud, P., 1976. Experimental and theoretical approach of the production and transformation of organic matter in a semi-enclosed basin. In: Proceedings of the 10th European Symposium on Marine Biology. Ed. by G. Persoone \& E. Jaspers. University Press, Wetteren, 2, 231-245.

Glansdorff, P. \& Prigogine, I., 1974. Thermodynamic theory of structure, stability and fluctuations. Wiley-Interscience, London, $306 \mathrm{pp}$.

Levin, S. A., 1976. Population dynamic models in heterogeneous environments. Ann. Rev. Ecol. Syst. 7, 287-310.

Thom, R., 1972. Structural stability and morphogenesis. Benjamin, Reading, Mass., 362 pp.

- 1974. Modèles mathématiques de la morphogénèse. Collection 10/18, UGE, Paris, 319 pp.

Turing, A. M., 1952. The chemical basis of morphogenesis. Proc. R. Soc., Lond. (B) 237, 37-72.

Author's address: Dr. D. Dubois

University of Liège

Institute of Mathematics (Biomathematics)

15 , avenue des Tilleuls

B-4000 Liège

Belgium 